\title{
Mechanical and Thermal Properties of Poly(urethane urea) Nanocomposites Prepared with Diamine-Modified Laponite
}

\author{
Joe-Lahai Sormana, ${ }^{1,2}$ Santanu Chattopadhyay, ${ }^{1,3}$ and J. Carson Meredith ${ }^{1}$ \\ ${ }^{1}$ School of Chemical \& Biomolecular Engineering, Georgia Institute of Technology, Atlanta, GA 30332-0100, USA \\ ${ }^{2}$ Rohm \& Haas, Chemicals LLC, Spring House Technical Center, Spring House, PA 19477-0904, USA \\ ${ }^{3}$ Rubber Technology Centre, Indian Institute of Technology Kharagpur, Kharagpur 721302, India
}

Correspondence should be addressed to J. Carson Meredith, carson.meredith@chbe.gatech.edu

Received 20 May 2008; Accepted 25 August 2008

Recommended by Alan Fuchs

\begin{abstract}
Nanocomposites based on segmented poly(urethane urea) were prepared by reacting a poly(diisocyanate) with diamine-modified Laponite-RD nanoparticles that served as a chain extender. The nanocomposites were prepared at a constant $\mathrm{NH}_{2}$ to $\mathrm{NCO}_{\mathrm{mole}}$ ratio of 0.95 , while varying the fraction of diamine-modified Laponite relative to the free diamine chain extender. Compared to neat poly(urethane urea), all nanocomposites showed increased tensile strength and elongation at break. As Laponite loading increased, tensile properties passed through a maximum at a particle concentration of 1 mass $\%$, at which a $300 \%$ increase in tensile strength and $40 \%$ increase in elongation at break were observed. A maximum in urea and urethane hard-domain melting endotherms was also observed at this Laponite loading. Optimal mechanical and thermal properties coincided with a minimum in the size of the inorganic Laponite phase. Nanocomposites containing diamine-modified Laponite had higher tensile strengths than those with nonreactive monoamine-modified Laponite or diamine-modified Cloisite.
\end{abstract}

Copyright ( $\odot 2008$ Joe-Lahai Sormana et al. This is an open access article distributed under the Creative Commons Attribution License, which permits unrestricted use, distribution, and reproduction in any medium, provided the original work is properly cited.

\section{INTRODUCTION}

The synthesis and characterization of polymer composites with nanometer-size silicates have become an extremely active research area, and significant improvements in mechanical, thermal, and barrier properties have been shown relative to the pristine polymers. Many studies have illuminated the difficulties of achieving nanometric dispersion of silicates in polymers, especially for layered silicates $[1,2]$. These difficulties arise apparently from weak intermolecular interactions between the silicate and the polymer, relative to the strong interactions between silicate layers.

Synthetic Laponite is a silicate with well-controlled dimensions $(1 \times 25 \mathrm{~nm}$ disc $)$ that forms less strongly-layered structures than those found in natural silicates. Laponite is readily modified with surfactants due to its complete exfoliation in water. A number of studies have demonstrated physical blending as well as chemical incorporation of Laponite in polymers [3-12]. The addition of functionalized (reactive) surfactants to Laponite, followed by incorporation into nanocomposites has not been reported extensively until recently $[8,12-15]$.

Despite the industrial importance of poly(urethane ureas) (PUUs) as specialty engineering elastomers, few PUUbased nanocomposites have been demonstrated [16-19]. PUU elastomers are block copolymers consisting of alternating rubbery (soft) and crystalline urea (hard) segments that provide physical cross-links between soft segments. The mechanical and thermal properties of PUUs depend on the size, crystallinity, and interconnectivity of the hard domains, as well as mixing of hard segments in the soft segment phase [20-23]. Depending on the ratio of hard and soft segments, and reaction temperature, the hard domain size can approach nanoscale dimensions on the order of $50 \mathrm{~nm}$ to $100 \mathrm{~nm}$. While PUU elastomers have excellent elastic properties, they are limited by the melting of hard domains at temperatures greater than $\sim 270^{\circ} \mathrm{C}$. One avenue for improving these properties is by dispersing inorganic nanoparticles. Due to the complex morphology of PUUs, the question arises how the presence of nanoparticles will affect the PUU morphology, crystallinity, and hydrogen bonding 
[24]. PUU nanocomposites with improved mechanical and barrier properties have been prepared by physically mixing organically modified montmorillonite with presynthesized PUU in solution [19]. The use of nanoparticles as participants in the chemical reaction to produce PUU networks has also received attention $[17,20-23,25,26]$. Studies have also reported the preparation of polyurethane (nonurea) nanocomposites with the nanoparticle as a reacting component of the chain-extender [27-31] or the prepolymer [32, 33]. These nanocomposites showed partial intercalation and significant improvement in tensile strength and elongation at break.

This paper reports on the surface modification of Laponite-RD particles with amine functional groups and their use in preparing Laponite-PUU nanocomposites. We describe an ion-exchange process for coating the particles with organic diamines, resulting in a free amine group tethered to the surface. These are characterized and compared to nanocomposites prepared with monoamine-coated Laponite (no free amine groups) and nanocomposites made with diamine-modified Cloisite-Na (montmorillonite). The relationship between Laponite dispersion, PUU structure, as well as mechanical and thermal properties is discussed.

\section{EXPERIMENTAL METHODS}

\subsection{Particle modification}

Laponite-RD and Cloisite-Na, having cationic exchange capacities of 75 mequiv/100 g and 92.6 mequiv/100 g, respectively, were obtained from Southern Clay Products Inc. (Gonzales, Tex, USA), and used as received. Laponite $\left(\mathrm{Na}^{+}{ }_{0.7}\left[\left(\mathrm{Si}_{8} \mathrm{Mg}_{5.5} \mathrm{Li}_{0.3}\right) \mathrm{O}_{20}(\mathrm{OH})_{4}\right]^{0.7-}\right)$ is a mica-type synthetic hectorite that hydrates in water to give a homogenous dispersion of exfoliated nanometric disks $(1 \mathrm{~nm} \times$ 25-30 nm) [34]. Cloisite-Na is a natural montmorillonite clay with a lateral size of about 100-1000 nm. Trimethylene glycol di- $p$-aminobenzoate (Air Products and Chemicals Inc., Allentown, Pa, USA, Versalink 740-M, 98.2\% purity, $314 \mathrm{~g} / \mathrm{mol}$ ) and 4-benzylaniline (Lancaster Synthesis, Ward Hill, M, USA, 98\% purity, $183 \mathrm{~g} / \mathrm{mol}$ ) were treated with excess concentrated $\mathrm{HCl}$ to form ammonium salt. For the remainder of this paper, trimethylene glycol di- $p$ aminobenzoate and 4-benzylaniline will be referred to as diamine and monoamine, respectively. The excess $\mathrm{HCl}$ was allowed to evaporate under ambient conditions for several days, resulting in a dry salt. The dried monoamine and diamine salts were crushed in a mortar and washed 3 times with tetrahydrofuran (VWR, ACS grade) to remove all untreated amines. The tetrahydrofuran (THF) was dried over molecular sieve to remove water prior to use. The purified ammonium salts were dried and characterized with FTIR and TGA.

The modification process involved a cation-exchange reaction between ammonium salt and cations present naturally on the silicate surface, as indicated in Figure 1(a). Three different exchange reactions were performed: (I) monoamine salt and Laponite-RD, (II) diamine salt and Laponite-RD, and (III) diamine salt and Cloisite-Na.

\subsubsection{Exchange reaction I}

Six grams of Laponite-RD were added to $600 \mathrm{~g}$ of deionized water and stirred with a magnetic stirring bar under ambient conditions for approximately 30 minutes. The clear Laponite-RD dispersion was slowly added to a solution of excess monoamine salt (twice the exchange capacity of Laponite-RD) in water. The mixture was stirred with a magnetic stirring bar at room temperature for 24 hours. The monoamine-modified Laponite-RD (MAML) particles were collected by centrifuge and repeatedly washed and centrifuged with DI water until halide anions were not detected. The presence of halide anions was monitored by adding $\mathrm{AgNO}_{3}$ to the supernatant solution after centrifuge to form a white $\mathrm{AgCl}$ precipitate. The final washed particles were dried under ambient conditions for several days, subsequently crushed in a mortar, and screened with a 325 mesh sieve.

\subsubsection{Exchange reactions /I and III}

The procedure for exchange reaction I was repeated with minor alterations. Both exchange reactions II and III were carried out at $60^{\circ} \mathrm{C}$. In exchange reaction III, Cloisite- Na was dispersed in hot water at $60^{\circ} \mathrm{C}$, prior to addition to the diamine salt solution. Exchange reactions II and III produced diamine modified Laponite-RD (DAML) and diamine modified Cloisite- $\mathrm{Na}(D A M C)$, respectively. The organic surface content of the modified particles was determined by the difference in weight between pure and modified particles from thermogravimetric analysis in the temperature range $150-1000^{\circ} \mathrm{C}$.

\subsection{Synthesis of poly(urethane urea)-silicate nanocomposites}

PUU nanocomposites were prepared by first dispersing modified particles in THF, followed by adding prepolymer to give solutions with 30 mass $\%$ prepolymer and 0.5 to 0.3 mass $\%$ particle. The prepolymer was toluenediisocyanate-(tetramethylene glycol) prepolymer (Air Products and Chemicals Inc., Allentown, Pa, USA, AIRTHANE PET-90A, $M_{w} \bullet 2350$, NCO functionality $=2.0$, mass $\%$ NCO $=3.58 \%$ ). All reactions, unless otherwise stated, were carried out at 95 mole\% of the stoichiometric amount of diamine needed to react with all the isocyanate groups present in the prepolymer. The diamine chain extender needed to achieve the desired stoichiometry was added from a 30 mass $\%$ stock solution in THF. PUU/DAML nanocomposites were prepared at different DAML weight fractions to optimize DAML concentration in the nanocomposites. The reacting mixture was stirred with a magnetic stirring bar for several minutes and the solution was coated on a Piranha cleaned silicon wafer (Silicon Inc., Boise, Idaho, USA) using a knife-edge coater $[35,36]$. Curing took place directly following film casting, in a vacuum oven at $90^{\circ} \mathrm{C}$ for 6 hours. For comparison purposes, both neat PUU and PUU nanocomposites using MAML and DAMC were prepared using the same procedures, with appropriate substitution of 


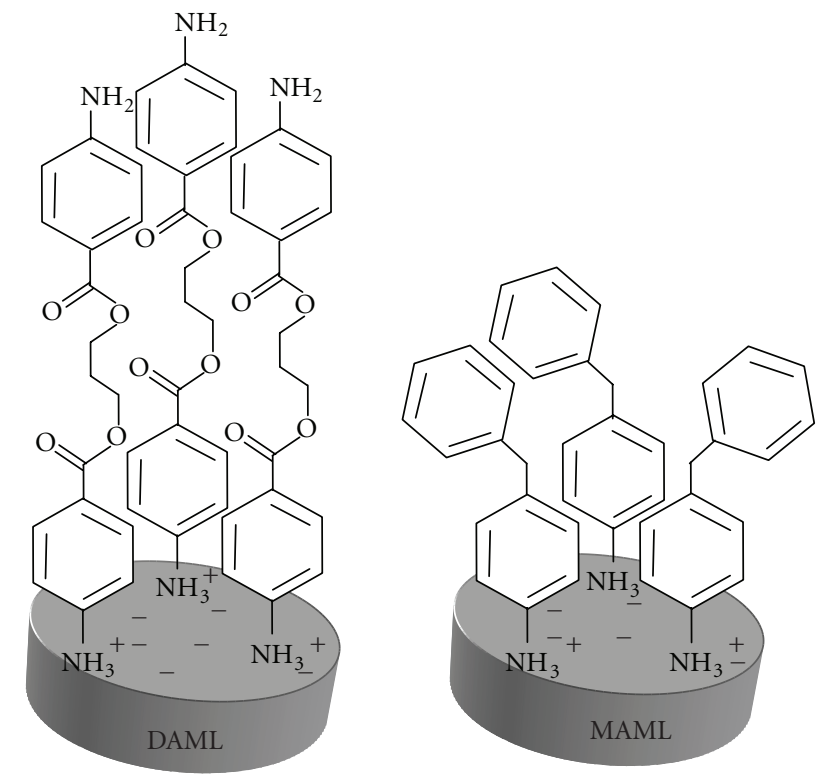

(a)

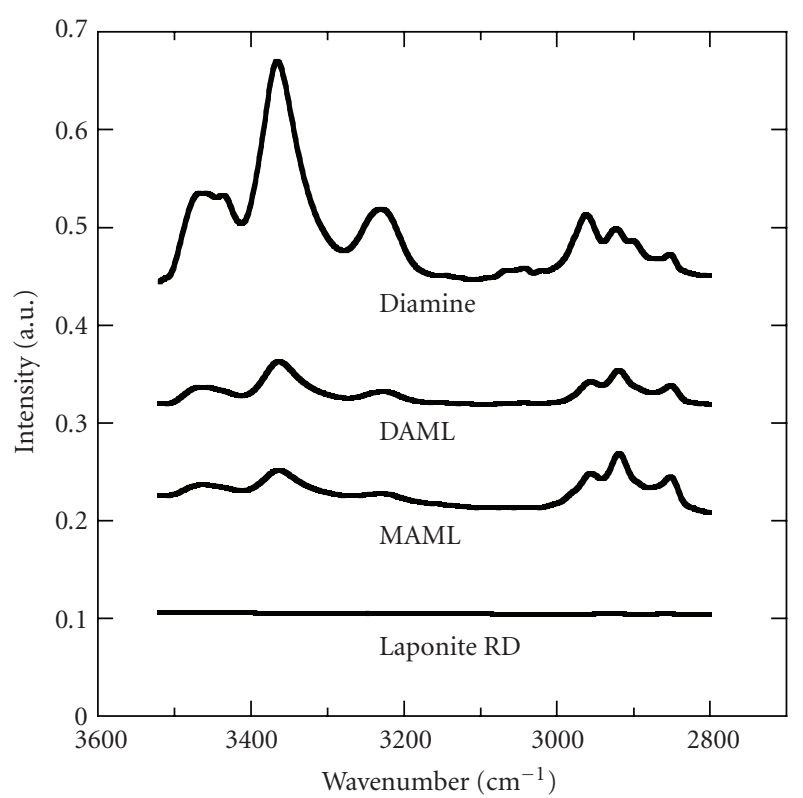

(b)

Figure 1: (a) Schematic illustrating chemical structure of modified Laponites. Structures are not drawn to scale and only one side of modification is indicated. DAML = diamine-modified Laponite and MAML = monoamine-modified Laponite. (b) FT-IR spectra in the $\mathrm{N}-\mathrm{H}$ stretch region of Laponite-RD particles before and after modification with (a) diamine and (b) monoamine.

starting materials. All nanocomposites were optically clear and indistinguishable to the naked eye from neat PUU.

\subsection{Characterization}

All nanocomposite film thicknesses were measured by visible-near infrared interferometry (Stellar Net EPP 2000) [37]. Thermogravimetric analysis (TGA) was carried out at a heating rate of $10^{\circ} \mathrm{C} / \mathrm{min}$ in a nitrogen atmosphere from $30^{\circ} \mathrm{C}$ to $1000^{\circ} \mathrm{C}$ (TA Instruments Model TGA-Q500). FTIR spectra of monoamine, diamine, Laponite- $\mathrm{RD}$, CloisiteNa, MAML, DAML, DAMC, and nanocomposite films were recorded at a resolution of $4 \mathrm{~cm}^{-1}$ under ambient condition using a Bruker IRscope II connected to a Bruker IFS 66/V spectrometer. The sample area was purged with dry air to prevent the appearance of atmospheric water bands in the spectra. All spectra were corrected for thickness and peak analysis software package (Peakfit 4.11, Jandel Scientific) was used to resolve the bands in the carbonyl $\left(v=1630-1760 \mathrm{~cm}^{-1}\right)$ region to obtain the relative ratios of the peak areas, assuming Gaussian peak shapes. Wideangle X-ray diffraction (WAXD) spectra of the unmodified (Laponite-RD and Cloisite-Na) and modified particle (MAML, DAML, and DAMC) powders were recorded using a PANanalytical X'Pert diffractometer with nickel-filtered $\mathrm{CuK} \alpha(\lambda=0.15406 \mathrm{~nm})$ radiation in a sealed tube, operated at $40 \mathrm{kV}$ and $200 \mathrm{~mA}$ at a scan rate of $6 \mathrm{deg} / \mathrm{min}$. The WAXD spectra of nanocomposite films, as prepared above, were collected with the same instrument with the incident beam normal to the film plane. The gallery spacing of the silicates, $d$, was determined using Bragg's equation: $n \lambda=2 d \sin (\theta)$. Mechanical properties of pure PUU and PUU nanocompos- ite films were determined using a commercial uniaxial tensile apparatus (INSTRON 5842) at a strain rate of $30 \mathrm{~mm} / \mathrm{min}$. Differential scanning calorimetry (DSC) measurements of the nanocomposites were performed on a Seiko DSC220C at a heating rate of $5^{\circ} \mathrm{C} / \mathrm{min}$ over the temperature range of -90 to $320^{\circ} \mathrm{C}$ in a nitrogen atmosphere. Transmission electron microscopy (TEM) was performed using a JEOL JEM-100CX II TEM at an acceleration voltage of $100 \mathrm{kV}$. Samples for TEM were prepared by coating onto a PELCO Formvar support film, stabilized with carbon on 200-mesh copper grid (Ted Pella, Inc., Redding, Calif, USA). The grid-coated samples were annealed at the same conditions as all others.

\section{RESULTS AND DISCUSION}

\subsection{Particle modification}

Figure 1(b) compares FT-IR spectra of diamine-modified particles to the neat diamine and unmodified Laponite- $\mathrm{RD}$ in the N-H stretch region. These spectra confirm the successful modification of Laponite and indicate the presence of free primary amine groups that can serve as chain extenders for isocyanates in poly(urethane urea) synthesis. FT-IR results for diamine modified Cloisite-Na (DAMC) and monoaminemodified Laponite (MAML) gave a similar indication of successful modification, not shown. In particular, free amine functionalities are not detected in the MAML FTIR spectra, as expected.

TGA was used to estimate the amount of monoamine or diamine tethered to the particles, shown in Figure 2. The organic amines contributed nearly 20 mass $\%$ of the DAML, $17 \%$ of the DAMC, and $10 \%$ of the total weight of 


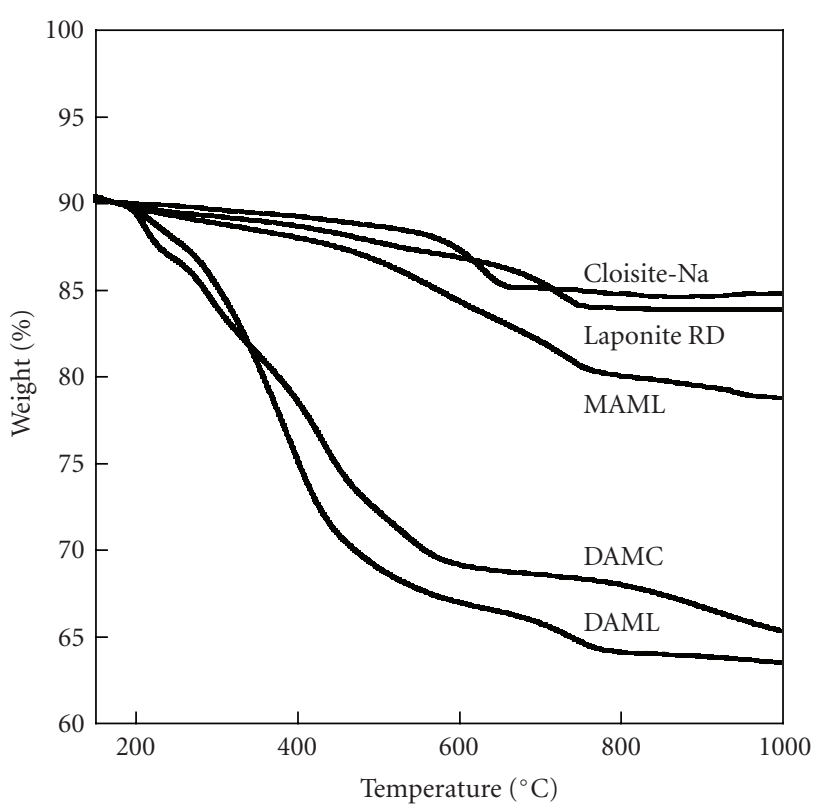

FIGURE 2: TGA curves of unmodified and modified silicates.

MAML. The pure monoamine and diamine (TGA graphs not included) showed complete decomposition in this temperature range. The reported CEC of Laponite, $75 \mathrm{mmol} / 100 \mathrm{~g}$, can be used to estimate a capacity of 19 mass $\%$ diamine at full exchange (see Supplementary Material available online at doi:10.1155/2008/869354), in close agreement with the TGA result of $20 \%$. However, the diamine is predicted to comprise $22 \%$ of the modified Cloisite mass. The lower TGAdetermined value (17 mass\%) suggests incomplete exchange with Cloisite. The significantly lower mass fraction amine on the MAML is due primarily to the lower molecular mass of the monoamine relative to the diamine. Complete exchange is predicted to result in a monoamine mass fraction of $12 \%$, not far from the observed value of $10 \%$. These quantities were used to determine the amount of excess diamine needed for the desired poly(urethane urea) stoichiometry.

WAXD powder spectra for unmodified and modified Laponite and Cloisite-Na are presented in Figure 3. The WAXD of unmodified Laponite-RD shows a single peak centered at $2 \theta=6.5 \AA\left(d_{001}=13.6 \AA\right)$, which is consistent with previously reported values $[38,39]$ and the absence of surfactant contaminant in the as-supplied material (also absent in FTIR Figure 1(b)). In the two modified Laponite diffraction patterns, a broad set of overlapping peaks from $1.5^{\circ}<2 \theta<9^{\circ}$ is observed. The breadth and presence of overlapping subpeaks at angles above and below $d_{001}$ suggest that a complex structure exists in the modified Laponite. It is difficult to specify precisely the structures present from the WAXD data. The low-angle peaks suggest that modification results in gallery expansion (intercalation of amine modifier) relative to neat Laponite. However, higherangle peaks suggest that some unmodified clay remains. The diffraction pattern for Cloisite-Na has a peak centered about $2 \theta=7.5^{\circ}$ ( $d_{001}$ spacing of $11.7 \AA$ ). The modified Cloisite- Na particle, DAMC, shows a single diffraction peak at $2 \theta=4.5^{\circ}$

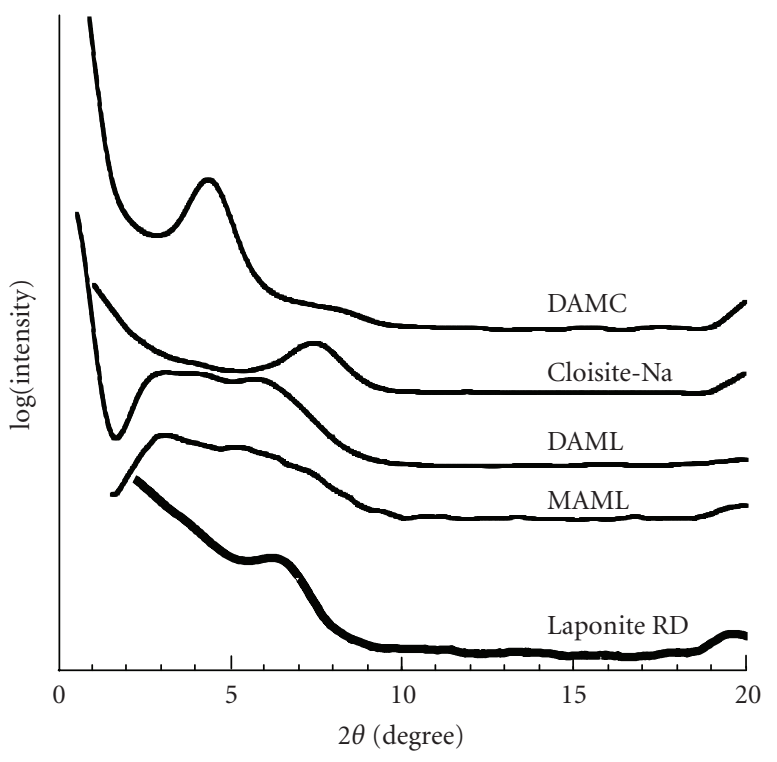

FIGURE 3: WAXD spectra of unmodified and modified Laponite-RD and Cloisite-NA silicate powders.

$\left(d_{001}=19.6 \AA\right)$, which indicates an expansion of the gallery spacing due to intercalated diamine.

\subsection{Nanocomposites}

Uniaxial tensile stress-strain curves for pure PUU and PUU nanocomposites with different amounts of DAML are presented in Figure 4 and the extracted mechanical properties are summarized in Figure 5 and Table 1. Both the tensile strength and elongation at break increased with DAML concentration up to a maximum at 1\% DAML. Interestingly, the modulus and shape of the stress-strain curve for the DAML nanocomposites are indistinguishable from those of pure PUU up to strains of $\approx 300 \%$. This suggests the mechanism by which DAML increases strength becomes active only at high elongations, for example, enhanced strain crystallization. The simultaneous increase in both tensile strength and elongation at break has been reported for montmorillonite nanocomposites with polyurethanes $[29,30]$. Ni et al. observed a two-fold increase in both tensile strength and elongation for poly(tetramethylene glycol)-based PUUs chain extended with a diamine-modified montmorillonite at $5 \%$ particle loading [26].

The amine moieties on diamine-modified Laponite compete with free-(dissolved) amine chain extender to form urea linkages during network formation. To investigate whether this competition leads to a significant effect on the urea morphology, we used FTIR and DSC to investigate $\mathrm{H}$ bonding and thermal properties of the hard domains, respectively. PUU hydrogen bonding, especially the bidentate ureaurea bonds, is critical to maintaining the phase-separated morphology and mechanical strength of hard domains [23, 37, 40]. FT-IR spectra of neat and nanocomposite PUU in the carbonyl region (shown in Supplementary Figure 1) indicate that free and hydrogen-bonded peaks of neat PUU 
TABLE 1: Mechanical properties and endotherms of PUU nanocomposites.

\begin{tabular}{|c|c|c|c|c|c|c|c|}
\hline $\begin{array}{l}\text { Nanocomposite } \\
\text { composition } \\
\text { (mass\% Silicate) }\end{array}$ & $\begin{array}{c}\mathrm{NH}_{2} \text { from particle }{ }^{\mathrm{a}} \\
(\text { mole } \%)\end{array}$ & $\begin{array}{c}\text { Tensile strength } \\
(\mathrm{MPa})\end{array}$ & $\begin{array}{l}\text { Elongation at break } \\
(\%)\end{array}$ & $\begin{array}{l}\text { Modulus } E_{100}{ }^{\mathrm{b}} \\
\quad(\mathrm{MPa})\end{array}$ & $\begin{array}{l}T_{g}{ }^{\mathrm{c}} \\
\left({ }^{\circ} \mathrm{C}\right)\end{array}$ & $\begin{array}{c}\text { Endo }^{\mathrm{d}} 1 \\
\text { Peak, } T\left({ }^{\circ} \mathrm{C}\right)\end{array}$ & $\begin{array}{c}\text { Endo }{ }^{\mathrm{d}} 2 \\
\text { Peak, } T\left({ }^{\circ} \mathrm{C}\right)\end{array}$ \\
\hline Neat PUU & 0 & $16.7 \pm 1.7$ & $501 \pm 34$ & $11.1 \pm 1.2$ & -72 & 192 & 274 \\
\hline 0.5 mass $\%$ DAML & 0.8 & $33.0 \pm 3.9$ & $604 \pm 17$ & $11.7 \pm 0.9$ & -70 & 192 & 270 \\
\hline 1 mass\% DAML & 1.7 & $48.8 \pm 1.4$ & $716 \pm 40$ & $11.6 \pm 1.0$ & -70 & 197 & 265,277 \\
\hline 1.5 mass $\%$ DAML & 2.6 & $26.4 \pm 2.7$ & $608 \pm 37$ & $10.4 \pm 1.4$ & -69 & 192 & 273 \\
\hline 3 mass\% DAML & 5.4 & $16.0 \pm 2.1$ & $642 \pm 35$ & $10.9 \pm 0.8$ & -68 & 188 & 277 \\
\hline 1 mass $\%$ MAML & 0 & $22.1 \pm 3.0$ & $734 \pm 21$ & $13.2 \pm 1.4$ & -68 & 189 & 271 \\
\hline 1 mass $\%$ DAMC & 1.7 & $28.4 \pm 0.7$ & $751 \pm 36$ & $10.3 \pm 0.6$ & -71 & 189 & 285 \\
\hline
\end{tabular}

${ }^{\mathrm{a}} \mathrm{Mole} \%-\mathrm{NH}_{2}$ groups contributed by particle out of total $-\mathrm{NH}_{2}$ moles in sample.

${ }^{b}$ Error represents a $95 \%$ confidence level from at least 3 samples.

${ }^{c}$ Soft segment glass transition temperature.

${ }^{\mathrm{d}}$ Endo means endothermic.

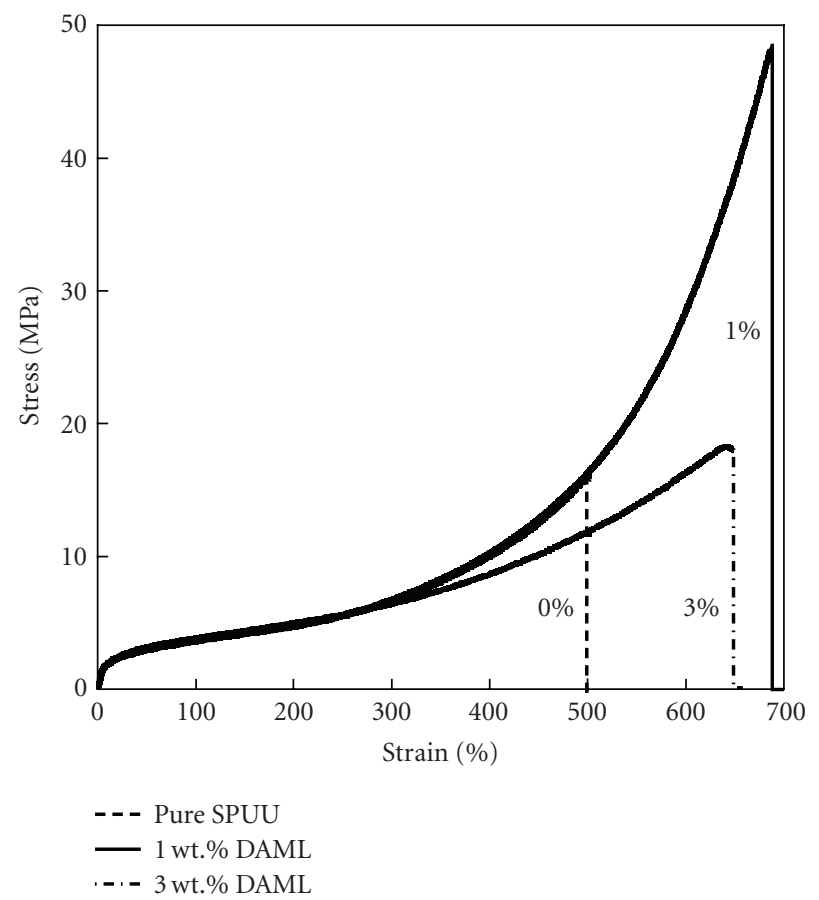

Figure 4: Stress-strain curves for pure PUU and PUU nanocomposites prepared with DAML at 1 and 3 wt.\% DAML.

are essentially identical to those found in the nanocomposites. The fractions of urea- and urethane-specific hydrogen bonding (shown in Supplementary Figure 1) were estimated from the carbonyl absorbances. The fraction of ordered and disordered urea and urethane-involved hydrogen bonds for all nanocomposites were statistically indistinguishable from one another and from the neat PUU.

Table 1 summarizes data from DSC thermographs (DSC traces are shown in Supplementary Figures 2 and 3). The soft segment glass transition temperatures of all nanocomposites are nearly identical to the glass transition temperature of pure PUU. This suggests that the dispersed silicates do not have a significant effect on the soft phase-free volume. A common feature of all the thermographs is the presence of

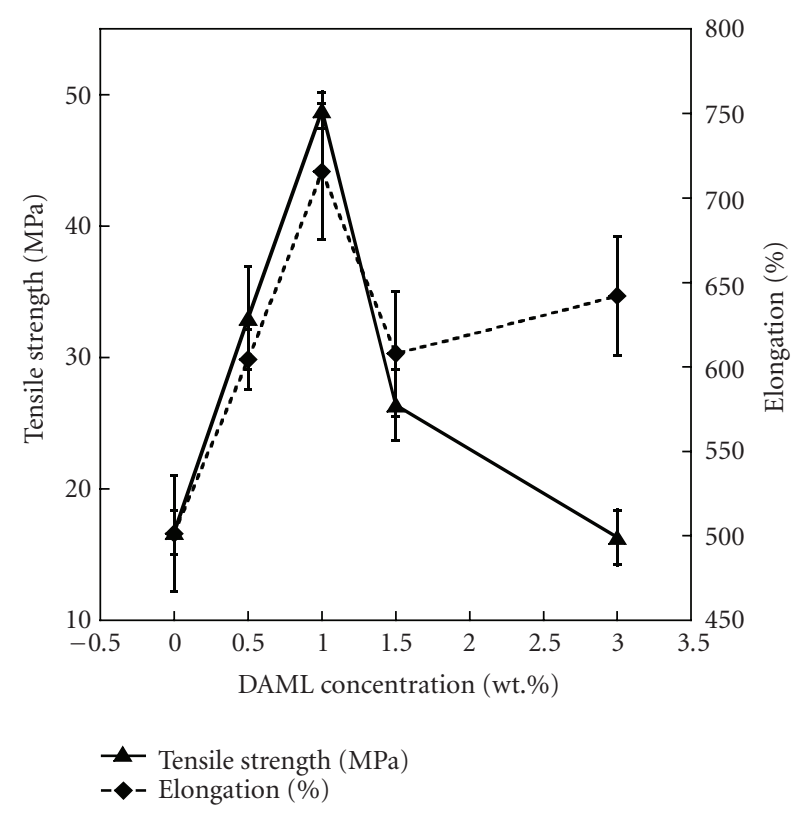

FIGURE 5: Mechanical properties of PUU-DAML nanocomposites prepared at different DAML concentrations.

two primary melting endotherms: one at about $190^{\circ} \mathrm{C}$ and another at about $270^{\circ} \mathrm{C}$. The high-temperature endotherm at $270^{\circ} \mathrm{C}$ represents melting of the crystalline urea hard domains, while the endotherm at $190^{\circ} \mathrm{C}$ is associated with melting of urethane containing hard domains. The pure PUU shows endotherms at 190 and $273^{\circ} \mathrm{C}$, with a slight shoulder peak at $288^{\circ} \mathrm{C}$. As DAML is added, the width, location, and number of peaks in the $270^{\circ} \mathrm{C}$ range change, whereas the $190^{\circ} \mathrm{C}$ endotherm shifts location slightly but does not split into multiple peaks. The 1\% DAML sample clearly shows two distinct peaks in the urea hard-domain endotherm, at $265^{\circ} \mathrm{C}$ and $277^{\circ} \mathrm{C}$, above and below the pure PUU endotherm of $273^{\circ} \mathrm{C}$. In addition, the $1 \%$ sample also shows $\mathrm{a}+5^{\circ} \mathrm{C}$ shift of the urethane endotherm, the only sample that showed an increase in this endotherm. The appearance of multiple endotherms in neat PU and 


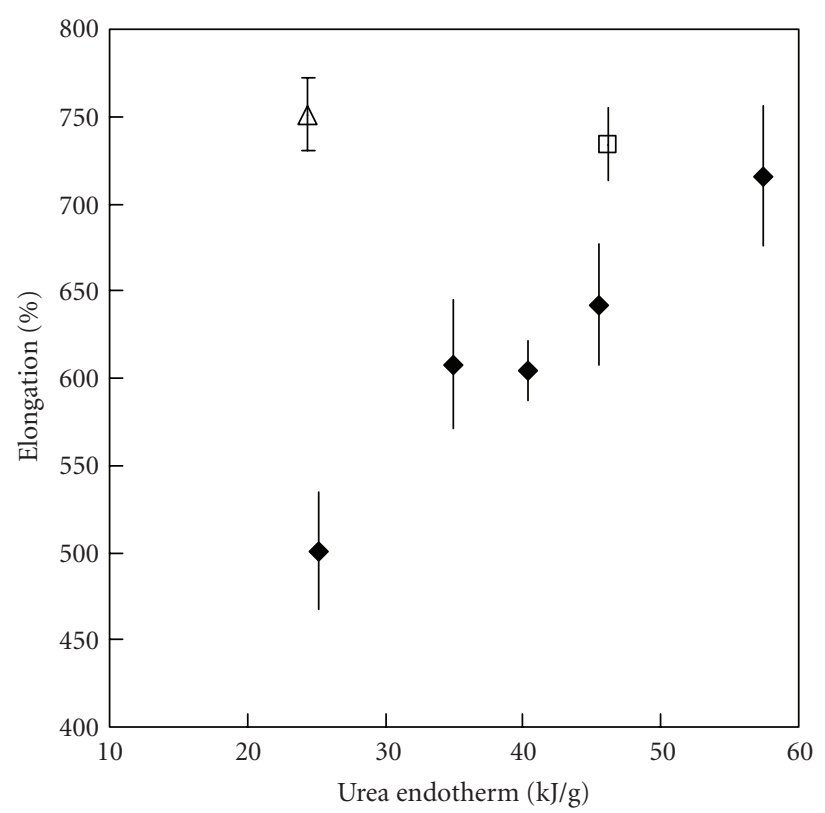

- DAML

$\square \quad 1 \%$ MAML

$\triangle 1 \%$ DAMC

(a)

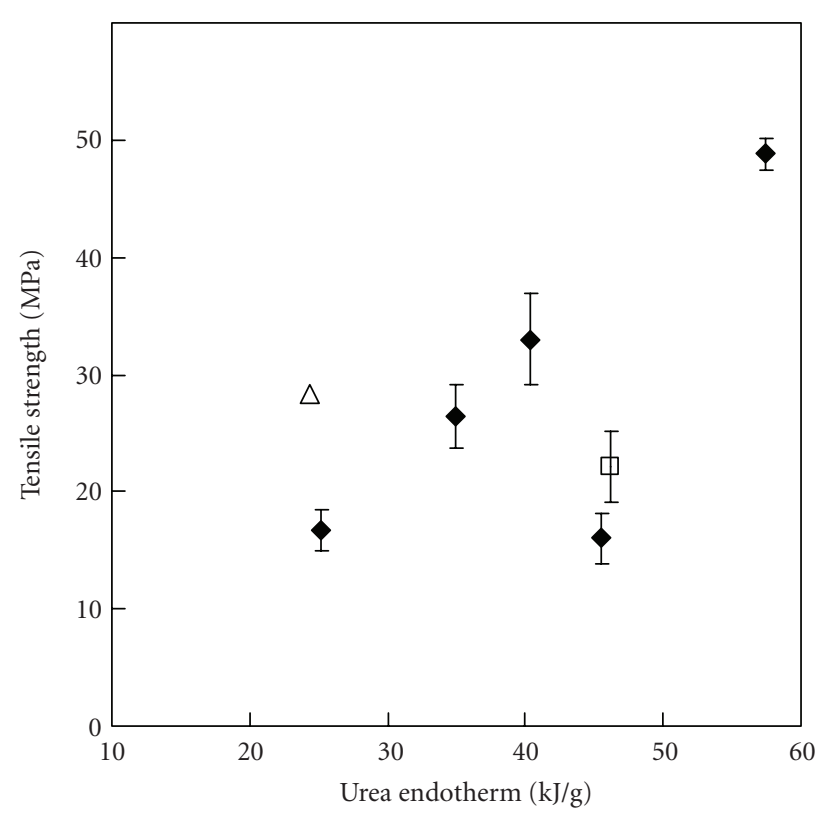

$\checkmark$ DAML

$\square \quad 1 \%$ MAML

$\triangle 1 \%$ DAMC

FIGURE 6: Elongation-at-break and tensile strength versus values of the urea endotherm for all nanocomposite samples.

PUU has been interpreted by others as signifying the presence of urea crystal lamellae with different thicknesses or crystallites with different polymorphism [22]. Similar endotherm increases and splitting have been observed in other nanocomposite-PU studies $[17,41]$. Tien and Wei [17] reported that diamine-modified montmorillonite PU nanocomposites showed a maximum melting endotherm at 1 mass $\%$ nanoparticle, which returned close to the unmodified endotherm value at 5 mass $\%$ particle.

Figure 6 shows a comparison of elongation and break and tensile strength versus values of the urea endotherm for all nanocomposite samples. The strongest correlation is between elongation and urea endotherm, which is linear for the DAML samples. On the contrary, elongation is not correlated linearly with the urethane endotherm, but rather passes through a minimum. Still the highest elongation, for the $1 \%$ DAML, did coincide with the highest urethane endotherm value. Both the urea and urethane crystallization endotherms have very similar correlations with tensile strength. All samples with the exception of the 3\% DAML show a linear correlation between tensile strength and both endotherms. The 3\% DAML sample shows a lower tensile strength than that predicted by the trend of the other samples with the urea endotherm.

WAXD spectra for the pure and nanocomposite PUUs (Supplementary Figure 4) showed no diffraction peaks in any of the nanocomposites. While the absence of peaks is consistent with exfoliation of silicate layers in the PUU nanocomposites, this cannot be taken as unambiguous evidence. There is, for example, the possibility of aggregates consisting of randomly-oriented particles, especially considering the low-aspect ratio of Laponite. Representative TEM micrographs of PUU and PUU-DAML nanocomposites prepared with $0 \%, 1 \%$, and $3 \%$ DAML are presented in Figure 7. The micrograph of the unmodified PUU (Figure 7(a)) shows no characteristic features except for faint outlines of the hard-domain semicontinuous phase. Nanocomposites prepared with 1 mass\% DAML contained features resembling circular discs, with a diameter of about 20-30 nm (Figure 7(b)), distributed nonuniformly throughout the sample (Figure $7(\mathrm{c})$ ). The DAML phase in the TEM images appears as if it were oriented preferentially in the direction of film spreading, although anisotropy cannot be confirmed unambiguously from our sample preparation method. However, anisotropic particle distributions have been observed for PEO-Laponite films prepared by spreading from aqueous solution $[9,11]$. Those authors showed that the platelets become aligned in the film plane as the network collapses during film drying [9, 11]. Although we cannot assign the particle geometry (disc, sphere, cylinder) or confirm the presence of exfoliation unambiguously, the shape and size of the inorganic content in the 1\% DAML samples are consistent with the Laponite diameter. In contrast, the TEM of the 3 mass $\%$ DAML sample indicates an aggregated inorganic phase with size much larger than single Laponite. In fact, at 3\% DAML, diameters range from $60 \mathrm{~nm}$ to $120 \mathrm{~nm}$ with a median of $80 \mathrm{~nm}$, as shown in Figure 7(c).

The data presented here indicate a strong correlation between the size and dispersion of the inorganic phase, 


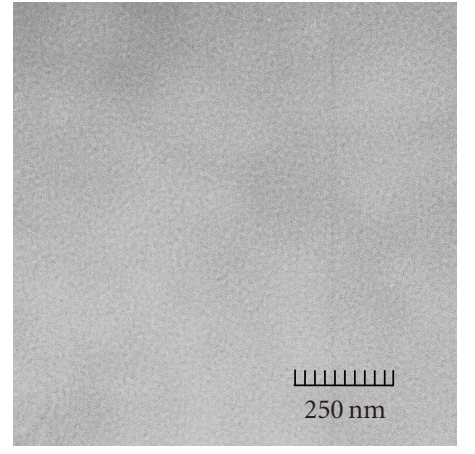

(a)

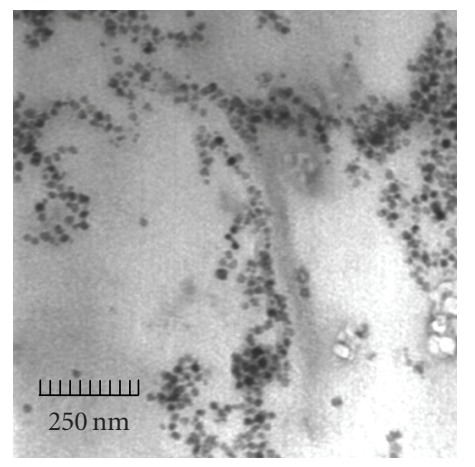

(b)

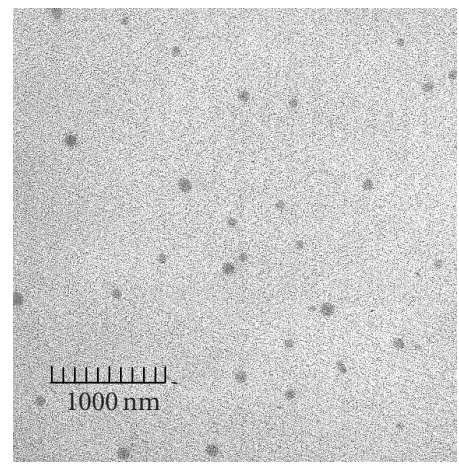

(c)

FIgURE 7: Representative TEM micrographs of (a) unmodified PUU and DAML-PUU nanocomposites containing (b) $1 \mathrm{wt} \%$ DAML and (c) 3 wt.\% DAML.

the changes in hard segment endotherms and the PUU nanocomposite mechanical properties.

It is apparent that at $1 \%$ DAML, the inorganic phase has a size on the order of $25 \mathrm{~nm}$ diameter, and that at $3 \%$ DAML, the size increases to $80 \pm 20 \mathrm{~nm}$. Due to the sample preparation and resolution of the TEM images, we cannot definitively conclude whether or not the Laponite is exfoliated into single discs. However, there is clearly a definitive increase in the dispersed size of the inorganic phase (about 4 -fold in diameter) in going from 1\% to 3\% DAML. The larger combined surface area of the Laponite phase at $1 \%$ DAML may expose the PUU to more confined surfacetethered urea groups than at 3\% DAML.
The loss in strength in the 3\% DAML sample is correlated with the increase in the size of the inorganic phase, compared to the $1 \%$ DAML samples. Also correlated is the urea hard-domain endotherm that was shifted to a maximum temperature at $1 \%$ DAML and returned to values close to the neat PUU hard-domain crystallization at 3\% DAML. None of these properties (size, strength, endotherms) were related to changes in the fraction of hydrogen bonds in urethane or urea groups. These correlations suggest that ordering induced by the tethered ureas colocated within a thin molecular plane on the Laponite might lead to improved mechanical and thermal properties. Under this scenario, urea crystallites tethered directly to Laponite might be expected to align easier and form larger domains than the free bulk urea segments. However, it is also possible that the nanoparticles may simply act as a hindrance to disordering and melting as temperature is increased [17]. Both scenarios are consistent with our thermal and mechanical observations but cannot be distinguished using the current data.

\section{CONCLUSIONS}

Segmented poly(urethane urea) nanocomposites were prepared using commercial Laponite and Cloisite silicates modified with reactive diamine and nonreactive monoamine groups. A 3-fold increase in tensile strength is observed with $1 \%$ by mass of diamine-modified Laponite, relative to unmodified Laponite. This is associated with a marked increase in PUU urea melting endotherm. As the loading of diamine-modified Laponite increases from 1\% to 3\% by mass, the size of Laponite inorganic phase increases dramatically, along with a return of urea hard segment endotherms and nanocomposite mechanical properties to values near unmodified PUU. Work is ongoing toward using high-resolution and orientation-dependent TEM and WAXD define precisely the size, shape, and dispersion of the Laponite phase.

\section{ACKNOWLEDGMENTS}

Support from Arkema Group Inc. is gratefully acknowledged. We thank Professor Chris Jones for use of TGA, Professor Haskell Beckham for use of DSC, and Professor Sankar Nair for use of WAXD.

\section{REFERENCES}

[1] R. Krishnamoorti, "Strategies for dispersing nanoparticles in polymers," MRS Bulletin, vol. 32, no. 4, pp. 341-347, 2007.

[2] G. Wegner, M. M. Demir, M. Faatz, et al., "Polymers and inorganics: a happy marriage?" Macromolecular Research, vol. 15, no. 2, pp. 95-99, 2007.

[3] E. R. Kleinfeld and G. S. Ferguson, "Rapid, reversible sorption of water from the vapor by a multilayered composite film: a nanostructured humidity sensor," Chemistry of Materials, vol. 7, no. 12, pp. 2327-2331, 1995.

[4] H.-C. Lee, T.-W. Lee, T.-H. Kim, and O. O. Park, "Fabrication and characterization of polymer/nanoclay hybrid ultrathin multilayer film by spin self-assembly method," Thin Solid Films, vol. 458, no. 1-2, pp. 9-14, 2004. 
[5] W. Loyens, P. Jannasch, and F. H. J. Maurer, "Poly(ethylene oxide)/Laponite nanocomposites via melt-compounding: effect of clay modification and matrix molar mass," Polymer, vol. 46, no. 3, pp. 915-928, 2005.

[6] D. F. Schmidt, F. Clément, and E. P. Giannelis, "On the origins of silicate dispersion in polysiloxane/layered-silicate nanocomposites," Advanced Functional Materials, vol. 16, no. 3, pp. 417-425, 2006.

[7] A. Senillou, N. Jaffrezic, C. Martelet, and S. Cosnier, "A laponite clay-poly(pyrrole-pyridinium) matrix for the fabrication of conductimetric microbiosensors," Analytica Chimica Acta, vol. 401, no. 1-2, pp. 117-124, 1999.

[8] K. M. Dean, S. A. Bateman, and R. Simons, "A comparative study of UV active silane-grafted and ion-exchanged organoclay for application in photocurable urethane acrylate nanoand micro-composites," Polymer, vol. 48, no. 8, pp. 2231-2240, 2007.

[9] A. Dundigalla, S. Lin-Gibson, V. Ferreiro, M. M. Malwitz, and G. Schmidt, "Unusual multilayered structures in poly(ethylene oxide)/laponite nanocomposite films," Macromolecular Rapid Communications, vol. 26, no. 3, pp. 143-149, 2005.

[10] W.-G. Hou, W.-A. Zhao, and D.-X. Li, "Synthesis and properties of polystyrene/laponite nanocomposites," Chinese Journal of Polymer Science, vol. 22, no. 5, pp. 459-462, 2004.

[11] M. M. Malwitz, P. D. Butler, L. Porcar, D. P. Angelette, and G. Schmidt, "Orientation and relaxation of polymerclay solutions studied by rheology and small-angle neutron scattering," Journal of Polymer Science Part B, vol. 42, no. 17, pp. 3102-3112, 2004.

[12] J. Wang, P. A. Wheeler, W. L. Jarrett, and L. J. Mathias, "Synthesis and characterization of dual-functionalized laponite clay for acrylic nanocomposites," Journal of Applied Polymer Science, vol. 106, no. 3, pp. 1496-1506, 2007.

[13] C. Konn, F. Morel, E. Beyou, P. Chaumont, and E. BourgeatLami, "Nitroxide-mediated polymerization of styrene initiated from the surface of laponite clay platelets," Macromolecules, vol. 40, no. 21, pp. 7464-7472, 2007.

[14] N. Negrete-Herrera, J.-L. Putaux, L. David, and E. BourgeatLami, "Polymer/laponite composite colloids through emulsion polymerization: influence of the clay modification level on particle morphology," Macromolecules, vol. 39, no. 26, pp. 9177-9184, 2006.

[15] N. Negrete-Herrera, J.-L. Putaux, L. David, F. De Haas, and E. Bourgeat-Lami, "Polymer/laponite composite latexes: particle morphology, film microstructure, and properties," Macromolecular Rapid Communications, vol. 28, no. 15, pp. 1567-1573, 2007.

[16] C.-C. M. Ma, Y.-L. Huang, H.-C. Kuan, and Y.-S. Chiu, "Preparation and electromagnetic interference shielding characteristics of novel carbon-nanotube/siloxane/poly-(urea urethane) nanocomposites," Journal of Polymer Science Part B, vol. 43, no. 4, pp. 345-358, 2005.

[17] Y. I. Tien and K. H. Wei, "Thermal transitions of montmorillonite/polyurethane nanocomposites," Journal of Polymer Research, vol. 7, no. 4, pp. 245-250, 2000.

[18] H.-L. Wu, C.-C. M. Ma, Y.-T. Yang, H.-C. Kuan, C.-C. Yang, and C.-L. Chiang, "Morphology, electrical resistance, electromagnetic interference shielding and mechanical properties of functionalized MWNT and poly(urea urethane) nanocomposites," Journal of Polymer Science Part B, vol. 44, no. 7, pp. 1096-1105, 2001.

[19] R. Xu, E. Manias, A. J. Snyder, and J. Runt, "Low permeability biomedical polyurethane nanocomposites," Journal of
Biomedical Materials Research Part A, vol. 64, no. 1, pp. 114119, 2003.

[20] J. T. Garrett, J. Runt, and J. S. Lin, "Microphase separation of segmented poly(urethane urea) block copolymers," Macromolecules, vol. 33, no. 17, pp. 6353-6359, 2000.

[21] J. T. Garrett, C. A. Siedlecki, and J. Runt, "Microdomain morphology of poly(urethane urea) multiblock copolymers," Macromolecules, vol. 34, no. 20, pp. 7066-7070, 2001.

[22] J. A. Miller, S. B. Lin, K. K. S. Hwang, K. S. Wu, P. E. Gibson, and S. L. Cooper, "Properties of polyether-polyurethane block copolymers: effects of hard segment length distribution," Macromolecules, vol. 18, no. 1, pp. 32-44, 1985.

[23] C. B. Wang and S. L. Cooper, "Morphology and properties of segmented polyether polyurethaneureas," Macromolecules, vol. 16, no. 5, pp. 775-786, 1983.

[24] J. G.-I. Rodríguez, P. Carreira, A. García-Diez, D. Hui, R. Artiaga, and L. M. Liz-Marzán, "Nanofiller effect on the glass transition of a polyurethane," Journal of Thermal Analysis and Calorimetry, vol. 87, no. 1, pp. 45-47, 2007.

[25] R. Y. Kanna, H. J. Salacinski, J. De Groot, et al., "The antithrombogenic potential of a polyhedral oligomeric silsesquioxane (POSS) nanocomposite," Biomacromolecules, vol. 7, no. 1, pp. 215-223, 2006.

[26] P. Ni, J. Li, J. Suo, and S. Li, "Novel polyether polyurethane/clay nanocomposites synthesized with organicmodified montmorillonite as chain extenders," Journal of Applied Polymer Science, vol. 94, no. 2, pp. 534-541, 2004.

[27] C. Zilg, R. Thomann, R. Mülhaupt, and J. Finter, "Polyurethane nanocomposites containing laminated anisotropic nanoparticles derived from organophilic layered silicates," Advanced Materials, vol. 11, no. 1, pp. 49-52, 1999.

[28] T.-K. Chen, Y.-I. Tien, and K.-H. Wei, "Synthesis and characterization of novel segmented polyurethane/clay nanocomposites," Polymer, vol. 41, no. 4, pp. 1345-1353, 2000.

[29] J. Ma, S. Zhang, and Z. Qi, "Synthesis and characterization of elastomeric polyurethane/clay nanocomposites," Journal of Applied Polymer Science, vol. 82, no. 6, pp. 1444-1448, 2001.

[30] Y. I. Tien and K. H. Wei, "High-tensile-property layered silicates/polyurethane nanocomposites by using reactive silicates as pseudo chain extenders," Macromolecules, vol. 34, no. 26, pp. 9045-9052, 2001.

[31] A. Rehab and N. Salahuddin, "Nanocomposite materials based on polyurethane intercalated into montmorillonite clay," Materials Science and Engineering A, vol. 399, no. 1-2, pp. 368-376, 2005.

[32] S.-I. Lee, Y. B. Hahn, K. S. Nahm, and Y.-S. Lee, "Synthesis of polyether-based polyurethane-silica nanocomposites with high elongation property," Polymers for Advanced Technologies, vol. 16, no. 4, pp. 328-331, 2005.

[33] M. Tortora, G. Gorrasi, V. Vittoria, G. Galli, S. Ritrovati, and E. Chiellini, "Structural characterization and transport properties of organically modified montmorillonite/polyurethane nanocomposites," Polymer, vol. 43, no. 23, pp. 6147-6157, 2002.

[34] R. G. Avery and J. D. F. Ramsay, "Colloidal properties of synthetic hectorite clay dispersions. II. Light and small angle neutron scattering," Journal of Colloid and Interface Science, vol. 109, no. 2, pp. 448-454, 1986.

[35] J. C. Meredith, A. Karim, and E. J. Amis, "Combinatorial methods for investigations in polymer materials science," MRS Bulletin, vol. 27, no. 4, pp. 330-335, 2002.

[36] J. C. Meredith, A. P. Smith, A. Karim, and E. J. Amis, "Combinatorial materials science for polymer thin-film dewetting," Macromolecules, vol. 33, no. 26, pp. 9747-9756, 2000. 
[37] J.-L. Sormana and J. C. Meredith, "High-throughput discovery of structure-mechanical property relationships for segmented poly(urethane-urea)s," Macromolecules, vol. 37, no. 6, pp. 2186-2195, 2004.

[38] D. Kubies, R. Jérôme, and J. Grandjean, "Surfactant molecules intercalated in laponite as studied by $13 \mathrm{C}$ and 29Si MAS NMR," Langmuir, vol. 18, no. 16, pp. 6159-6163, 2002.

[39] B. S. Shemper, J.-F. Morizur, M. Alirol, A. Domenech, V. Hulin, and L. J. Mathias, "Synthetic clay nanocomposite-based coatings prepared by UV-Cure photopolymerization," Journal of Applied Polymer Science, vol. 93, no. 3, pp. 1252-1263, 2004.

[40] H.-L. Tyan, Y.-C. Liu, and K.-H. Wei, "Enhancement of imidization of poly(amic acid) through forming poly(amic acid)/organoclay nanocomposites," Polymer, vol. 40, no. 17, pp. 4877-4886, 1999.

[41] B. Finnigan, D. Martin, P. Halley, R. Truss, and K. Campbell, "Morphology and properties of thermoplastic polyurethane nanocomposites incorporating hydrophilic layered silicates," Polymer, vol. 45, no. 7, pp. 2249-2260, 2004. 

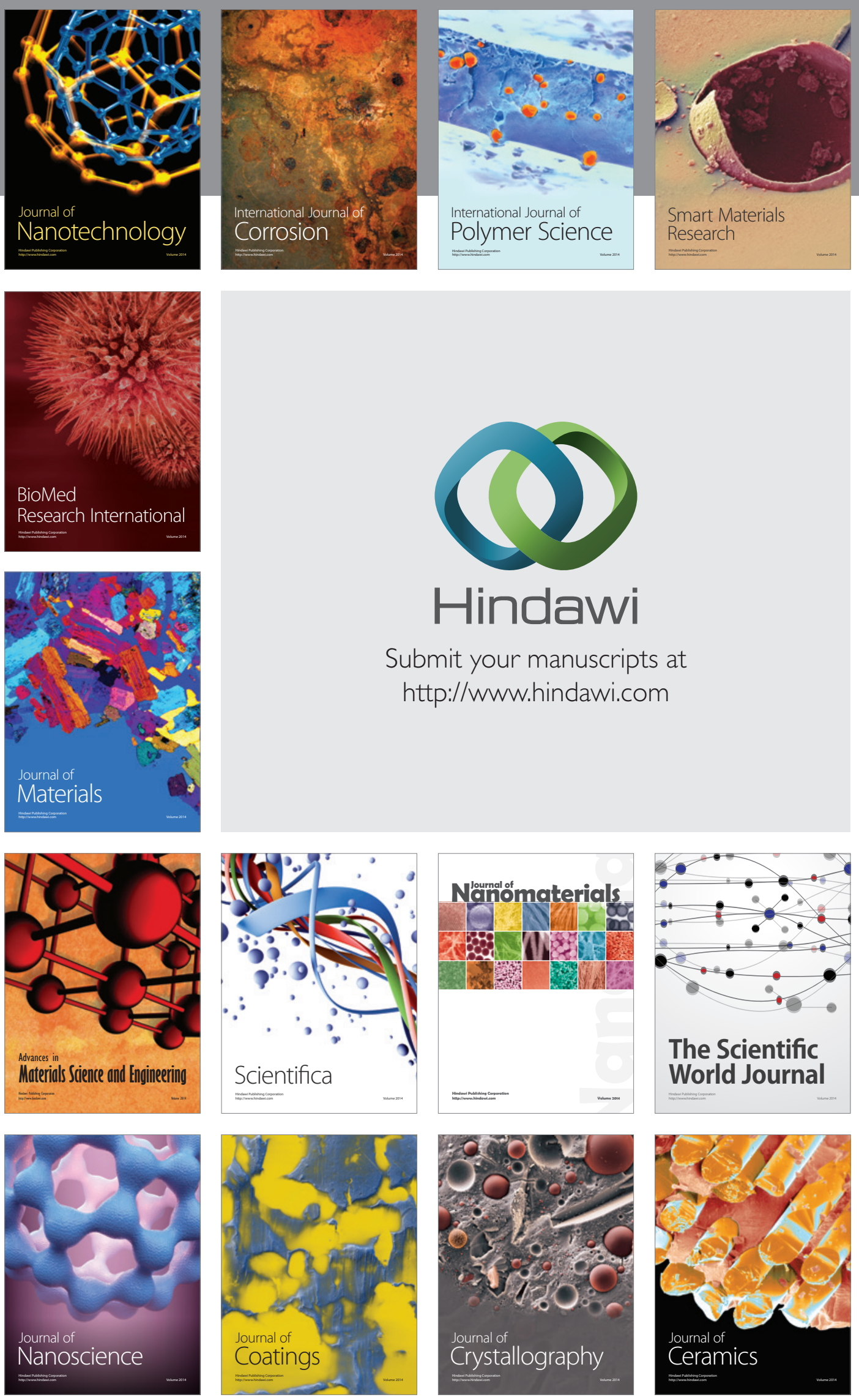

The Scientific World Journal

Submit your manuscripts at

http://www.hindawi.com

\section{World Journal}

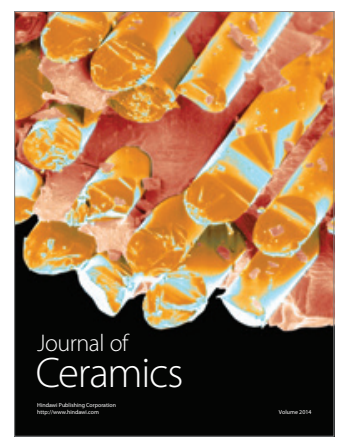

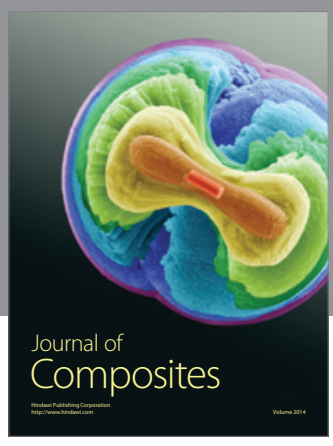
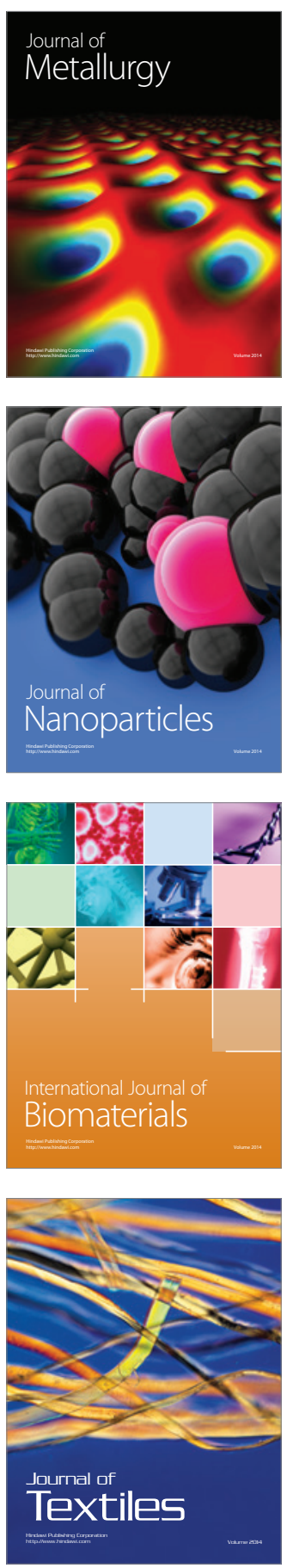